\title{
Motor Skill Learning Induces Changes in White Matter Microstructure and Myelination
}

\author{
Cassandra Sampaio-Baptista, ${ }^{1}$ Alexandre A. Khrapitchev, ${ }^{4}$ Sean Foxley, ${ }^{1}$ Theresa Schlagheck, ${ }^{1}$ Jan Scholz,,${ }^{1,6}$ \\ Saad Jbabdi, ${ }^{1}$ Gabriele C. DeLuca, ${ }^{2}$ Karla L. Miller, ${ }^{1}$ Amy Taylor, ${ }^{3}$ Nagheme Thomas, ${ }^{5}$ Jeffrey Kleim, ${ }^{5}$ Nicola R. Sibson, ${ }^{4}$ \\ David Bannerman, ${ }^{3}$ and Heidi Johansen-Berg ${ }^{1}$ \\ ${ }^{1}$ Oxford Centre for Functional MRI of the Brain and ${ }^{2}$ Neuropathology, Nuffield Department of Clinical Neurosciences, John Radcliffe Hospital, ${ }^{3}$ Department \\ of Experimental Psychology, and ${ }^{4}$ Cancer Research-UK/Medical Research Council Gray Institute for Radiation Oncology and Biology, Department of \\ Oncology, University of Oxford, Oxford OX1 2JD, United Kingdom, ${ }^{5}$ School of Biological and Health Systems Engineering, Arizona State University, Tempe, \\ Arizona 85287, and ${ }^{6}$ Mouse Imaging Centre, Hospital for Sick Children, Toronto, Ontario M5T 3H7, Canada
}

Learning a novel motor skill is associated with well characterized structural and functional plasticity in the rodent motor cortex. Furthermore, neuroimaging studies of visuomotor learning in humans have suggested that structural plasticity can occur in white matter (WM), but the biological basis for such changes is unclear. We assessed the influence of motor skill learning on WM structure within sensorimotor cortex using both diffusion MRI fractional anisotropy (FA) and quantitative immunohistochemistry. Seventy-two adult (male) rats were randomly assigned to one of three conditions (skilled reaching, unskilled reaching, and caged control). After $11 \mathrm{~d}$ of training, postmortem diffusion MRI revealed significantly higher FA in the skilled reaching group compared with the control groups, specifically in the WM subjacent to the sensorimotor cortex contralateral to the trained limb. In addition, within the skilled reaching group, FA across widespread regions of WM in the contralateral hemisphere correlated significantly with learning rate. Immunohistological analysis conducted on a subset of 24 animals (eight per group) revealed significantly increased myelin staining in the WM underlying motor cortex in the hemisphere contralateral (but not ipsilateral) to the trained limb for the skilled learning group versus the control groups. Within the trained hemisphere (but not the untrained hemisphere), myelin staining density correlated significantly with learning rate. Our results suggest that learning a novel motor skill induces structural change in task-relevant WM pathways and that these changes may in part reflect learning-related increases in myelination.

\section{Introduction}

We learn new motor skills throughout life, and this ability allows us to adapt to new environments and compensate for injury. Understanding the neurobiological basis for motor learning is important for both informing efforts to enhance learning and accelerating recovery from brain injury. The functional changes associated with motor learning are well described in both human and animal systems (Dayan and Cohen, 2011). In rats, learning a novel skilled reaching task is associated with well characterized

Received July 18, 2013; revised Oct. 15, 2013; accepted Nov. 4, 2013.

Author contributions: C.S.-B., J.S., J.K., N.R.S., D.B., and H.J.-B. designed research; C.S.-B., A.A.K., S.F., T.S., G.C.D., K.L.M., A.T., N.T., and D.B. performed research; C.S.-B., S.F., T.S., J.S., S.J., and H.J.-B. analyzed data; C.S.-B., A.A.K., S.F., J.S., S.J., G.C.D., K.L.M., A.T., N.T., J.A.K., N.R.S., D.B., and H.J.-B. wrote the paper.

This work was supported by the Wellcome Trust. C.S.-B. was the recipient of an FCT fellowship, and H.J.-B. was supported by a Wellcome Trust Senior Research Fellowship. We are grateful for support from the National Institute for Health Research Oxford Biomedical Research Centre based at Oxford University Hospitals National Health Service Trust and University of Oxford and to Carolyn Sloan for assistance with histology.

The authors declare no competing financial interests.

This article is freely available online through the J Neurosci Author Open Choice option.

Correspondence should be addressed to Prof. Heidi Johansen-Berg, Functional MRI of the Brain, John Radcliffe Hospital, Headington, 0xford 0X3 9DU, UK. E-mail: heidi.johansen-berg@ndcn.ox.ac.uk.

DOI:10.1523/JNEUROSCI.3048-13.2013

Copyright $\odot 2013$ Sampaio-Baptista et al.

This is an Open Access article distributed under the terms of the Creative Commons Attribution License (http://creativecommons.org/licenses/by/3.0), which permits unrestricted use, distribution and reproduction in any medium provided that the original work is properly attributed. functional reorganization of cortical motor maps, including expanded representation of the trained limbs (Kleim et al., 1998, 2004). This functional remapping is accompanied by a variety of structural changes, including synaptogenesis, increase in spine formation, and glial changes (Kleim et al., 2004; Xu et al., 2009).

The aforementioned studies have all focused on changes occurring in gray matter (GM) with motor learning. However, there is increasing evidence that learning may also influence white matter (WM) structure and function (Fields, 2008). Human studies provide some support for this in the motor domain. For example, learning to juggle, which has been shown previously to induce GM structural change in visual motion areas (Draganski et al., 2004), is also associated with increases in fractional anisotropy (FA), a measure of WM microstructure, in WM underlying cortical areas involved in eye-hand coordination (Scholz et al., 2009). However, because FA is modulated by several aspects of WM structure, such as myelination, axon diameter, axon density, and fiber organization (Beaulieu, 2002), it is not possible to pinpoint what structural event underlies a change in FA (Zatorre et al., 2012).

Animal studies provide a number of candidate mechanisms for learning-related changes in WM. For example, exposure to environmental enrichment during adulthood results in a higher number of unmyelinated and myelinated axons and glial cells (Markham et al., 2009; Zhao et al., 2012). A recent attempt to 
clarify which cellular events underlie changes in MRI measurements with a hippocampal-dependent spatial learning task found increases in FA in the rat corpus callosum as well as increases in myelin expression as measured by immunohistochemistry (Blumenfeld-Katzir et al., 2011). However, the hippocampus is one of the few brain structures that possess the capacity for adult neurogenesis in response to learning (Gould et al., 1999). Thus, WM changes here could be driven by new neurons establishing new efferent connections. Cortical neurogenesis has not been established, and thus studying tasks that induce cortical plasticity allows for assessment of WM plasticity without the generation of new neurons.

Here, we investigate WM plasticity associated with learning of a novel motor skill in rats by combining MRI and immunohistochemistry. MRI has the advantage of whole-brain coverage, thus offering insight as to where WM plasticity might be occurring and providing guidance to histology. Conversely, histology offers the possibility to validate MRI measurements and to shed light on the cellular events that underlie the measures obtained in human neuroimaging studies of motor learning.

\section{Materials and Methods}

Experiments were approved by the United Kingdom Home Office. Three separate batches of 24 (total $=72$ ), adult male Lister hooded rats $(4-5$ months old, 250-450 g) (Harlan) were housed in groups of three (one per experimental condition) in standard laboratory conditions under a $12 \mathrm{~h} \mathrm{light/dark}$ cycle at $20^{\circ} \mathrm{C}$ temperature and $40-70 \%$ humidity. All animals were given appropriate time to acclimatize after delivery, with ad libitum access to typical food and water. All animals were food controlled to $85-90 \%$ of their free-feeding weight (averaged over 3 consecutive days) 1 week before the start of the behavioral training. Weight was closely monitored to ensure that the animals weight did not fall below $85 \%$ of their original weight. Before training commenced and during food control, all animals were handled daily and provided with sucrose pellets in their home cage to habituate them to this new food type. All behavioral training and testing was performed during the light phase.

Animals were randomly assigned to one of three experimental conditions: (1) skilled reach (SR); (2) unskilled reach (UR); and (3) no training control condition (CC).

\section{Behavioral training}

The behavioral paradigm followed previously published guidelines on a single-pellet reaching task (Kleim et al., 2004).

Pretraining. Before behavioral training, all animals underwent pretraining sessions of $30 \mathrm{~min} / \mathrm{d}$ for $3 \mathrm{~d}$. A container filled with sucrose pellets ( $45 \mathrm{mg}$; Bioserv) was placed in front of the cage within easy reach. The rats were trained to reach into the container through the cage openings and retrieve pellets. The pretraining stopped when the animal retrieved 10 pellets or $30 \mathrm{~min}$ had passed. The animals were permitted to use either limb. The preferred limb was noted for each animal.

Training. After $3 \mathrm{~d}$ of pretraining, only the SR and UR animals were further trained on the single-pellet reaching task for 15 min daily for 11 consecutive days. The training sessions were conducted in a cage with just one opening and a tray with two small indentations centered on the edges of the opening, $1.3 \mathrm{~cm}$ away from the inside wall of the cage.

$S R$ condition. The pellet was placed in the indentation that could be reached by the preferred limb of the animal. The animal had five attempts to successfully grasp the pellet, which together constituted one trial. Successful reaches were scored when an animal grasped the pellet and guided it to its mouth without dropping it.

UR condition. To control for increased motor activity of the forelimbs, UR animals were placed in the same conditions as the SR group but the pellet was placed out of reach, preventing the animals from developing reaching/grasping skills. To keep the animals motivated, a pellet was dropped inside the cage periodically (every five attempts). On the last day of the experiment, the UR group was tested for reaching accuracy by putting the pellet within reach.
CC condition. The control animals did not receive any training after completion of the $3 \mathrm{~d}$ of pretraining, but they were handled daily, were weight monitored, and received a corresponding amount of sucrose pellets in their home cage.

\section{Behavioral measures and statistical analyses}

The accuracy score was calculated as follows: number of successful retrievals/total number of reaches. Number of total reaches corresponds to the sum of all reaches per day. Rate of learning was calculated as the slope of a fitted logarithmic model to the learning curve of each individual animal. A repeated-measures (RM) ANOVA was used to investigate improvement in accuracy scores over time in the SR group. A separate RM-ANOVA was run to investigate differences in total number of reaches between the SR and UR groups. To confirm the absence of skill learning in the UR group, accuracy scores on the final day of testing, when both groups were tested, were compared using a Student's $t$ test. Behavioral measures were correlated with histological and imaging measures as described below.

\section{Perfusion and brain preparation}

After training was completed, all animals were injected with $200 \mathrm{mg} / \mathrm{kg}$ sodium pentobarbital intraperitoneally and perfused transcardially with saline solution $(20 \mathrm{ml} / \mathrm{min})$ and newly prepared paraformaldehyde (PFA; 4\%). After perfusion, the brains were removed from the skull and stored in PFA until additional processing. For MRI acquisition, to keep the brains in a fixed position, brains were placed into Falcon tubes (50 $\mathrm{ml}$ ) in pairs, one brain above the other, and embedded in $2 \%$ agarose gel (Sigma).

\section{MRI acquisition}

Ex vivo brains were scanned overnight using a multichannel 7 T MRI scanner (Varian). Diffusion tensor imaging (DTI) scanning parameters were as follows: four diffusion averages; 30 diffusion encoding directions per average; spin-echo multislice (SEMS) diffusion sequence; eight images with no diffusion weighting; 40 slices; slice thickness, $0.5 \mathrm{~mm}$; field of view, $25 \times 50 \mathrm{~mm}$; matrix size, $96 \times 192$; resolution, $0.26 \times 0.26 \mathrm{~mm}$; $b=2000 \mathrm{~s} / \mathrm{mm}^{2}$.

\section{$M R$ processing and statistical analysis}

We performed analyses with the FSL (for FMRIB Software Library) package, version 4.1 (www.fmrib.ox.ac.uk/fsl). Brains were extracted from the gel with the Brain Extraction Tool. DTI data were analyzed with the FMRIB Diffusion Toolbox. FA and mean diffusivity (MD) were estimated from the original DTI data with dtifit and then analyzed using a modified version of Tract-Based Spatial Statistics (TBSS; Smith et al., 2006). The trained limb was consistently aligned across animals. The FA images were first aligned with linear transformation (six degrees of freedom and then 12 degrees of freedom) to an existing rat brain template to generate the study-specific template. All FA maps were aligned with linear and nonlinear transformations to this template and averaged to generate the mean FA image, from which the WM skeleton was extracted. The skeleton was thresholded at an FA value of 0.36 . This threshold was chosen based on our previous pilot studies using similar imaging protocols in different animals (Scholz, 2010) to reliably contain major tracts that can be accurately aligned across individuals. Finally, the FA values of the tract centers (i.e., maximum FA values) were projected onto the skeleton for each rat brain and fed into statistical analysis. MD values for the same voxels were also projected into the skeleton.

Nonparametric permutation testing with a cluster-forming threshold of $t>2$ and 5000 permutations were used to determine corrected $p$ values. Clusters with a corrected significance of $p<0.05$ were deemed significant. We tested for differences between the SR group and the control groups (UR and CC conditions) and for whole-brain correlations between FA and learning rate within the SR group.

\section{Histology}

A subset of 24 brains (all from one batch; $n=8$ per experimental group, one contralateral hemisphere from one animal in the SR group was damaged) was processed for immunohistochemistry. Brain tissue was sectioned into blocks containing the regions of interest (ROIs) and 
A



B

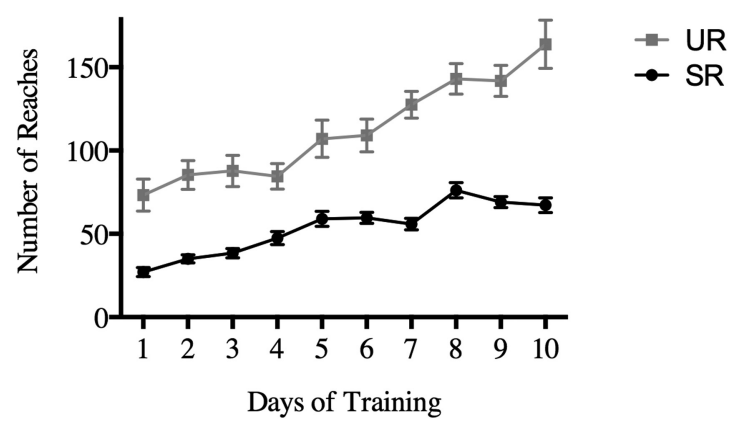

Figure 1. Behavioral results. A, Average accuracy scores for SR animals for all training days (black) and UR rats for the day 11 test (gray) $(n=24)$. $\boldsymbol{B}$, Average number of reaches per day for SR (black) and UR (gray) ( $n=24$ animals per group). Error bars represent SEM.

embedded in paraffin. Blocks were sectioned at $6 \mu \mathrm{m}$ using a rotary microtome (Leica). Sections were dewaxed, rehydrated, immersed in a solution of $10 \% \mathrm{H}_{2} \mathrm{O}_{2}\left(25 \mathrm{ml}\right.$ of $\mathrm{H}_{2} \mathrm{O}_{2}$ in $225 \mathrm{ml}$ of $0.1 \mathrm{M}$ PBS, pH 7.4), placed in citrate buffer, $\mathrm{pH} 6$, and heated in a microwave. The slides were mounted into sequenzas (Shandon Sequenza; Thermo Fisher Scientific) and washed twice with Tris-buffered saline/Tween 20 (TBS/T, pH 7.6; REAL EnVision kit detection system K5007; Dako). The anti-myelin basic protein (MBP; Millipore) primary antibody was mixed with TBS/T (1:500 dilution), pipetted onto the cover plates, and incubated for $1 \mathrm{~h}$ at room temperature. After washing the sections with TBS/T, the secondary antibody (REAL EnVision kit detection system K5007; Dako) was added and incubated for $40 \mathrm{~min}$. Finally, 3,3'-diaminobenzidine (volumes according to the Dako EnVision protocol) was added for $5 \mathrm{~min}$. Sections were subsequently dehydrated, cleared with histoclear, and mounted with coverslips with DPX.

\section{Histology measures and statistical analyses}

Myelin staining intensities were quantified with StereoInvestigator software (MBF Bioscience). Our primary hypothesis concerned the WM subjacent to M1 contralateral to the trained forelimb because M1 is the region that undergoes GM plasticity after training on this task (Kleim et al., 1998, 2002, 2004; Xu et al., 2009). For each section, the ipsilateral WM subjacent to M1 was used as control. Additional ROIs were defined according to FA results.

To correct for any light bias, background correction was performed by using an area without tissue within each slide. Images for all sections were acquired on the same day under the same light conditions, and ROIs were manually drawn by an experimenter blind to the experimental conditions.

S1 and M1 were identified and manually drawn in coronal sections, and ROIs were defined according to a rat atlas (Paxinos and Watson, 2007). The subjacent WM was segmented according to cortical region colocalization and luminance values extracted. Luminance measures are between 0 (black) and 254 (white). Low luminance reflects higher stain intensity.

We hypothesized that MBP staining intensity would be higher for the SR group compared with the controls and tested this with a Student's $t$ test (one-tailed). Correlations between staining intensity and learning rate were tested using Pearson's correlation for each hemisphere (twotailed). Comparison of the strength of correlations was made using Fisher's $r$ to $z$ transformation.

\section{Results}

\section{Behavioral results}

For the SR group, an RM-ANOVA on reaching accuracy scores over $11 \mathrm{~d}$ of testing showed a significant effect of day $\left(F_{(10,143.272)}=\right.$ $25.340, p<0.001)$, confirming that accuracy improved over time as the rats learned the task (Fig. $1 A$ ).

To assess whether specific skill learning was greater in the SR group compared with the UR group, the UR group was tested on the skilled version of the task on day 11 . Their performance was significantly worse than the SR group performance on the same day $\left(t_{(46)}=3.379, p<0.001\right)$, confirming that skill learning is significantly greater in the SR group (Fig. $1 A$ ).

We wanted to rule out the possibility that increased motor activity in the SR group could explain any observed changes in brain structure, and so total number of reaches per day was recorded. RM-ANOVA on total reaches per day in both the UR and SR groups, over $10 \mathrm{~d}$ of training, revealed a main effect of group $\left(F_{(1,46)}=78.257, p=0.001\right)$, a main effect of day $\left(F_{(9,159.145)}=\right.$ $44.791, p<0.001)$, and an interaction between day and group $\left(F_{(9,159.145)}=15.042, p<0.001\right.$; Fig. $\left.1 B\right)$. In summary, the SR group reaches less overall as they need to make fewer reaches to retrieve a pellet (the UR animals are given a pellet after five reaches). Over time, both groups of animals learn that reaching results in pellets and so their number of reaches increases, but so too does the differential between the groups as the SR group becomes increasingly accurate on the task.

\section{Neuroimaging results}

We found significant differences in FA between the SR animals and the control groups (UR and CC; $p<0.05$, corrected; $n=24$ animals per group), underlying sensorimotor GM areas in the contralateral hemisphere to the trained paw (Fig. 2A). Significantly lower MD was found for the same comparison in areas that overlap the clusters of significant FA. Specifically, 71\% of the voxels in the MD cluster overlapped with the voxels in the FA cluster. Localization of specific fiber tracts is not possible with the current data resolution, but the significant clusters overlap with regions corresponding to the cingulum, external capsule, and corpus callosum (Paxinos and Watson, 2007).

Additionally, a positive correlation was found between FA and learning rate for the SR rats in the hemisphere contralateral to the trained paw in widespread areas of WM $(p<0.05$, corrected; Fig. 2B).

\section{Histology results}

MBP stain intensity was significantly higher for the SR group compared with both control groups (UR and CC; $t_{(21)}=1.779$, $p<0.05$, one-tailed) in the ROI that corresponds to the WM subjacent to M1, contralateral to the trained paw (Fig. $3 A, B$ ). MBP stain intensity was also significantly different between SR and UR animals in the same area (Fig. $3 A, B ; t_{(13)}=1.905, p<$ 0.05 , one-tailed). No significant differences between groups for the ipsilateral side to the trained paw were found nor for any other ROIs $\left(t_{(22)}=-0.203, p=0.421\right)$. 
Within the SR group, learning rate correlated with WM MBP staining intensity in the contralateral M1 (Pearson's $r=$ $-0.788, p<0.05$, two-tailed; Fig. 3C) but not the ipsilateral M1 (Pearson's $r=$ $0.138, p=0.744$, two-tailed; Fig. $3 D$ ), resulting in a significant difference between the two correlations $(z=-1.7, p<0.05)$.

We did not find any correlation between MBP and FA or MD.

\section{Discussion}

We studied a skilled unilateral reaching task that has been associated previously with significant functional remapping and structural remodeling within the rat forelimb motor cortex (Kleim et al., 2002; $\mathrm{Xu}$ et al., 2009). Using diffusion MRI, we were able to show for the first time that this task also induces structural changes in WM underlying the forelimb motor cortex. Parallel histological measures demonstrated increased myelin staining in these same regions.

Although a previous study has made similar observations after training on a hippocampal-dependent spatial navigation task (Blumenfeld-Katzir et al., 2011), the current study is the first to report experience-dependent WM plasticity after a cortical learning paradigm, during which neurogenesis is unlikely to occur. Therefore, the current findings suggest that WM structural plasticity, and growth of new myelin, can occur without the generation of new neurons.

Although there is a vast literature on both functional and structural changes in GM associated with motor skill learning (for review, see Dayan and Cohen, 2011), there has been far less focus on the potential for WM to play a role in acquisition of new motor skills. Our results suggest that structural changes in WM, and in particular increases in myelination, are associated with acquisition of new motor skills. Experiments in cell culture provide support for the concept of activity-dependent modulation of myelin (Demerens et al., 1996). Fine tuning the timing and integration of sensorimotor signals is an important step in skill learning (Serrien and Brown, 2003; Fields, 2008). Modulation of myelin thickness may be one route by which long-range synchronization of signals is regulated (Fields, 2008).

Furthermore, we found a positive correlation between learning rate and FA in the hemisphere contralateral to the trained paw in WM areas that comprise the external capsule, cingulum, corpus callosum, and internal capsule. Some of these WM regions overlap with the areas found to be significantly increased in FA and decreased in MD in the SR group. A correlation with behavior indicates that variation in FA in these regions might be caused

A represent SEM. ${ }^{*} p<0.05$.


Figure 2. A, TBSS between-group analysis revealed that SR animals have significantly higher FA than UR and CC animals in WM areas comprising the external capsule and cingulum colocalized with somatomotor $G M$ areas in the contralateral hemisphere to the trained paw. Right, Mean FA from within the significant cluster is plotted for each group to visualize the range of values. $\boldsymbol{B}$, TBSS within-group correlation analysis showed that, within the SR group, animals with steeper learning rates have higher FA in widespread WM areas. Right, Scatter plot of mean FA within the significant cluster versus learning rate for individual animals is displayed for visualization of the range of values. All clusters shown at $p<0.05$, corrected for multiple comparisons.


Figure 3. $\boldsymbol{A}$, Representative anti-MBP-stained sections. Scale bar, $200 \mu \mathrm{m}$. $\boldsymbol{B}$, SR animals have significantly higher MBP stain intensity than UR animals. C, Animals with higher MBP stain intensity in WM subjacent M1 contralateral to the trained paw learn the reaching task faster $(n=7)$. $\boldsymbol{D}$, No correlation with performance was found for the WM underlying M1 on the ipsilateral side $(n=8)$. Low luminance values reflect higher stain intensity. Vertical scales have been inverted for intuitive reading. Error bars

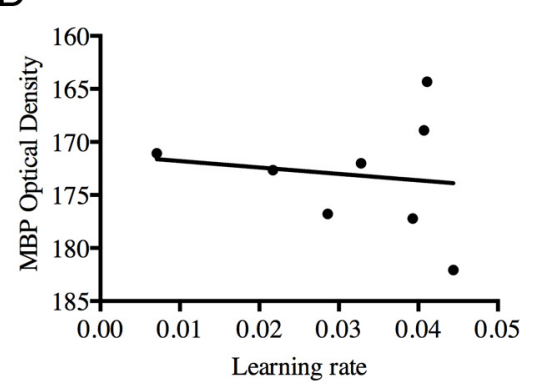

by the learning experience, but this correlation could also reflect preexisting inter-individual differences between animals, which in turn determine variations in skill learning behavior. Only a longitudinal design would be able to clearly establish a relationship between skill learning and FA change.

In accordance with the FA results, the histological findings also suggest that individual differences in WM structure had behavioral relevance. Specifically, we found that MBP expression in the hemisphere contralateral to the trained paw corre- 
lated with performance, whereas expression in the ipsilateral hemisphere did not. Myelin thickness is regulated by MBP, which indicates that myelin thickness might be involved in WM plasticity in response to learning (Martini and Schachner, 1997).

Despite the consistency in group differences detected in the neuroimaging and histological results, we did not find any correlations between measures of MBP expression and FA or MD. This might be attributable to the complexity of the diffusion parameters. FA is modulated by several WM characteristics, such as axon diameter, axon packing, and fiber organization (Beaulieu, 2002). A previous study that used a spatial learning task also found an increase in MBP expression, along with increases in FA, in corpus callosum, but did not report any correlation between the two measures (Blumenfeld-Katzir et al., 2011). It is possible that myelin is but one aspect of the WM that has undergone plasticity and FA is capturing several WM features, thus a correlation cannot be established. Furthermore, the sample size used for histology in the current study is relatively small; it is possible that a larger sample size would be more sensitive to a correlation between histological measures and FA or MD.

One limitation of the current study is that imaging was not performed before training and it is therefore possible that preexisting differences in brain structure could contribute to our findings. However, because animals were randomly assigned to different training conditions, we consider this unlikely. Nevertheless, future work using serial imaging would help to clarify the contribution of baseline differences in brain structure.

This work has implications for the interpretation of human imaging studies of the effects of learning on WM structure. Previous human neuroimaging studies have shown that FA changes in response to motor learning (Scholz et al., 2009), but the underlying cellular events were unknown (Zatorre et al., 2012). Consistent both with previous observations after hippocampaldependent spatial learning in rats (Blumenfeld-Katzir et al., 2011) and with demonstrations of correlations between FA and histological measures of myelin in a pathological context (Kolasinski et al., 2012), our findings suggest that at least some of the changes in FA with a cortical-dependent learning task could be explained by increases in myelination.

In conclusion, our results suggest that WM plasticity plays a hitherto overlooked role in the acquisition of new motor skills. This observation opens up new possibilities for predicting and assessing motor learning based on measures of WM structure. This is relevant in both the field of motor learning expertise (such as athletes or highly skilled professional groups) and the field of motor rehabilitation after brain damage, such as stroke.

\section{References}

Beaulieu C (2002) The basis of anisotropic water diffusion in the nervous system: a technical review. NMR Biomed 15:435-455. CrossRef Medline

Blumenfeld-Katzir T, Pasternak O, Dagan M, AssafY (2011) Diffusion MRI of structural brain plasticity induced by a learning and memory task. PLoS One 6:e20678. CrossRef Medline
Dayan E, Cohen LG (2011) Neuroplasticity subserving motor skill learning. Neuron 72:443-454. CrossRef Medline

Demerens C, Stankoff B, Logak M, Anglade P, Allinquant B, Couraud F, Zalc B, Lubetzki C (1996) Induction of myelination in the central nervous system by electrical activity. Proc Natl Acad Sci U S A 93:9887-9892. CrossRef Medline

Draganski B, Gaser C, Busch V, Schuierer G, Bogdahn U, May A (2004) Neuroplasticity: changes in grey matter induced by training. Nature 427 : 311-312. CrossRef Medline

Fields RD (2008) White matter in learning, cognition and psychiatric disorders. Trends Neurosci 31:361-370. CrossRef Medline

Gould E, Beylin A, Tanapat P, Reeves A, Shors TJ (1999) Learning enhances adult neurogenesis in the hippocampal formation. Nat Neurosci 2:260265. CrossRef Medline

Kleim JA, Barbay S, Cooper NR, Hogg TM, Reidel CN, Remple MS, Nudo RJ (2002) Motor learning-dependent synaptogenesis is localized to functionally reorganized motor cortex. Neurobiol Learn Mem 77:63-77. CrossRef Medline

Kleim JA, Hogg TM, VandenBerg PM, Cooper NR, Bruneau R, Remple M (2004) Cortical synaptogenesis and motor map reorganization occur during late, but not early, phase of motor skill learning. J Neurosci 24: 628-633. CrossRef Medline

Kleim JA, Barbay S, Nudo RJ (1998) Functional reorganization of the rat motor cortex following motor skill learning. J Neurophysiol 80:33213325. Medline

Kolasinski J, Stagg CJ, Chance SA, Deluca GC, Esiri MM, Chang EH, Palace JA, McNab JA, Jenkinson M, Miller KL, Johansen-Berg H (2012) A combined post-mortem magnetic resonance imaging and quantitative histological study of multiple sclerosis pathology. Brain 135:2938-2951. CrossRef Medline

Markham JA, Herting MM, Luszpak AE, Juraska JM, Greenough WT (2009) Myelination of the corpus callosum in male and female rats following complex environment housing during adulthood. Brain Res 1288:9-17. CrossRef Medline

Martini R, Schachner M (1997) Molecular bases of myelin formation as revealed by investigations on mice deficient in glial cell surface molecules. Glia 19:298-310. CrossRef Medline

Paxinos G, Watson C (2007) The rat brain in stereotaxic coordinates, 6th Ed. Amsterdam: Academic/Elsevier.

Scholz J (2010) Structural brain plasticity: Individual differences and changes with learning. $\mathrm{PhD}$ thesis, University of Oxford.

Scholz J, Klein MC, Behrens TE, Johansen-Berg H (2009) Training induces changes in white-matter architecture. Nat Neurosci 12:1370-1371. CrossRef Medline

Serrien DJ, Brown P (2003) The integration of cortical and behavioural dynamics during initial learning of a motor task. Eur J Neurosci 17:10981104. CrossRef Medline

Smith SM, Jenkinson M, Johansen-Berg H, Rueckert D, Nichols TE, Mackay CE, Watkins KE, Ciccarelli O, Cader MZ, Matthews PM, Behrens TE (2006) Tract-based spatial statistics: voxelwise analysis of multi-subject diffusion data. Neuroimage 31:1487-1505. CrossRef Medline

Xu T, Yu X, Perlik AJ, Tobin WF, Zweig JA, Tennant K, Jones T, Zuo Y (2009) Rapid formation and selective stabilization of synapses for enduring motor memories. Nature 462:915-919. CrossRef Medline

Zatorre RJ, Fields RD, Johansen-Berg H (2012) Plasticity in gray and white: neuroimaging changes in brain structure during learning. Nat Neurosci 15:528-536. CrossRef Medline

Zhao YY, Shi XY, Qiu X, Lu W, Yang S, Li C, Chen L, Zhang L, Cheng GH, Tang Y (2012) Enriched environment increases the myelinated nerve fibers of aged rat corpus callosum. Anat Rec (Hoboken) 295:999-1005. CrossRef Medline 